Pensamiento Crítico N. ${ }^{\circ}$ 13, pp. 71-90

\title{
Metodología de evaluación ex post
}

\author{
David Medianero Burga
}

\section{RESUMEN}

El presente artículo ofrece una síntesis de la metodología de evaluación ex post empleada por el autor en la evaluación del II Programa de Inversiones Sociales y de Infraestructura contra la Pobreza (en adelante, el Programa), ejecutado por el MEF, con apoyo financiero de la Corporación Andina de Fomento (CAF). El objetivo de dicha acción fue evaluar los niveles de pertinencia, eficacia, eficiencia, impacto y sostenibilidad de los proyectos de inversión pública conformantes del Programa. A tal efecto, el marco metodológico adoptado tomó en consideración que el objeto de una evaluación consiste en determinar si los proyectos generan los efectos deseados en las personas e instituciones a los cuales están dirigidos, de conformidad con los lineamientos establecidos en los documentos de pre inversión y evaluación ex ante aprobados por las instancias correspondientes. En general, la metodología utilizada en la presente evaluación está orientada a establecer conclusiones válidas respecto al desempeño de los proyectos en lo relativo a los criterios de pertinencia, eficacia, eficiencia, sostenibilidad e impacto. Esta metodología es replicada en situaciones similares, razón por la cual se brinda esta breve síntesis.

Palabras clave: Evaluación, eficiencia, productividad, metas, impacto. 


\title{
Pensamiento Crítico N. 13
}

\begin{abstract}
This article provides a synthesis of ex post evaluation methodology used by the author in the evaluation of the Second Social Investment Program Infrastructure and Poverty (hereinafter the Program), executed by the MEF, with financial support from the Corporación Andina de Fomento (CAF). The aim of this action was to assess the levels of relevance, effectiveness, efficiency, impact and sustainability of public investment projects making up the program. To this end, the methodological framework adopted took into consideration that the object of an evaluation is to determine if the projects generate the desired effects on individuals and institutions which are run in accordance with the guidelines established in the pre-investment documents and appraisal approved by the official agencies. In general, the methodology used in this evaluation is oriented to draw valid conclusions about the performance of projects with regard to the criteria of relevance, effectiveness, efficiency, sustainability and impact. This methodology may be replicated in similar situations, that is why this brief summary is provided.
\end{abstract}

Keywords: Evaluation, efficiency, productivity, goals, impact.

\section{Marco de la evaluación}

El objetivo del Programa fue contribuir con la política del Gobierno Nacional de lucha contra la pobreza a través de proyectos de inversión social y de infraestructura que permitieran mejorar las condiciones de vida de la población y propiciaran la generación de oportunidades de desarrollo económico y social. El Programa adoptó un enfoque de desarrollo humano integral, enmarcado en el proceso de descentralización del país. En el contrato de préstamo se asume que la ejecución de inversiones sociales y de infraestructura beneficiará a la población marginada del país, al favorecer objetivos específicos en sectores tales como agricultura, educación, energía, saneamiento, salud, transportes y vivienda. Igualmente, se espera que la concentración de inversiones en diferentes sectores de una misma región, tenga un impacto geográfico adecuado que favorezca el crecimiento económico y la inclusión social, fortaleciendo y profundizando el proceso de descentralización regional que implementa el Perú.

En la realización del estudio el marco metodológico adoptado parte de la consideración de que el objeto de la evaluación consiste en determinar si los proyectos han generado los efectos deseados en las personas e instituciones a los cuales estuvieron 


\section{David Medianero Burga}

dirigidos, de conformidad con los lineamientos establecidos en los documentos de pre inversión y evaluación ex ante aprobados por las instancias correspondientes. En tal perspectiva, se buscó determinar la medida en que los proyectos lograron generar los cambios deseados en la población objetivo y se buscará, asimismo, comparar, en la medida de lo posible, cuál habría sido la situación de la población si los proyectos no se hubieran ejecutado. Por otra parte, la evaluación permitió arribar a conclusiones fundamentales respecto al desempeño de los proyectos en lo relativo a los criterios de pertinencia, eficacia, eficiencia, sostenibilidad e impacto.

\section{Cadena de resultados}

En el contexto de la evaluación mencionada, se adoptó el enfoque de gestión por resultados, sustentado en el concepto de cadena de resultados, el cual, a su vez, toma como punto de partida el marco lógico de una intervención. Tomando como punto de partida el marco lógico de un proyecto, el propósito y demás objetivos concurrentes pueden ser visualizados y conceptualizados en forma interrelacionada de la siguiente manera:

- Los impactos se encuentran localizados en la filas del fin del marco lógico.

- Los efectos se encuentran localizados en la fila del propósito.

- Los productos, localizados en la fila de los componentes, se refieren a los productos entregados por el proyecto a la población beneficiaria o, en términos amplios, al país.

- Los procesos se encuentran localizados en la fila de las actividades. Están asociados a las actividades y se expresan en metas físicas y financieras.

Como se muestra en el Gráfico 2, el marco lógico establece la jerarquía de objetivos y sus correspondientes indicadores para los tres niveles de resultados: productos, efectos e impactos. Después de culminada la ejecución de un proyecto, debe realizarse el análisis de los resultados del mismo. En esta etapa, a través del marco lógico, se puede determinar la pertinencia, eficacia, eficiencia, coherencia y sostenibilidad del proyecto. Para ello será necesario relacionar entre sí las distintas filas y columnas del marco lógico, 


\section{Pensamiento Crítico N. 13}

especialmente aquellos casilleros relacionados a los objetivos del proyecto. En todos los casos, se espera que la evaluación proporcione a la institución ejecutora y al conjunto de involucrados del proyecto, información confiable que permita extraer lecciones sobre aspectos clave de la promoción del desarrollo que permita elevar la calidad de las futuras intervenciones.

Gráfico 1. Marco lógico y evaluación de resultados.

\begin{tabular}{c|l|l|l|l|l|}
\hline Objetivos & Indicadores & $\begin{array}{c}\text { Medios de } \\
\text { Verificación }\end{array}$ & Supuestos \\
\hline Resultados & Fin & $\begin{array}{l}\text { Indicadores } \\
\text { de impacto }\end{array}$ & & \\
\cline { 2 - 5 } & Propósito & $\begin{array}{l}\text { Indicadores } \\
\text { de efecto }\end{array}$ & & \\
\hline Procesos & Productos & $\begin{array}{l}\text { Indicadores } \\
\text { de producto }\end{array}$ & & \\
\hline Actividades & $\begin{array}{l}\text { Costos } \\
\text { (Insumos) }\end{array}$ & & \\
\hline
\end{tabular}

En el contexto de una evaluación ex post, el concepto de cadena de resultados es un elemento fundamental. Es un enfoque que tiene por fundamento la idea de que el empeño en alcanzar los resultados previstos debe guiar la estrategia de ejecución de los procesos. Para asegurar que se alcancen los resultados se debe supervisar permanentemente la ejecución del proyecto, comparando las metas planeadas con las metas efectivamente obtenidas, tanto a nivel físico-técnico como a nivel financiero. La cadena de resultados muestra la relación causal entre costos, actividades, productos, efectos directos y el impacto de largo plazo. La razón en que se sustenta la gestión basada en los resultados es que la estrategia de ejecución se debe centrar en el impacto, efectos directos y productos previstos, y que sobre esta base se deberán determinar las actividades y recursos necesarios para alcanzarlos.

En el contexto de un sistema de monitoreo y evaluación, se entiende por indicadores a los instrumentos utilizados para evaluar el grado de cumplimiento de los 


\section{David Medianero Burga}

resultados previstos. Asi, puede ser necesario analizar varios indicadores para evaluar si se ha logrado un determinado resultado. Como puede observarse en el Gráfico 2, existe una interacción entre los resultados y los procesos.

Los resultados son los logros esperados con la ejecución de los procesos en un orden sucesivo y de creciente importancia.

Los procesos denotan a las actividades que deben realizarse para la obtención de los resultados previstos, lo que implica el consumo de determinados recursos, los cuales en términos monetarios representan los costos del proyecto.

En general, en el marco del enfoque de gestión por resultados, se pueden identificar tres niveles de resultados: resultados de primer, segundo y tercer nivel; productos, efectos e impactos, respectivamente.

Gráfico 2. Cadena de resultados y evaluación.

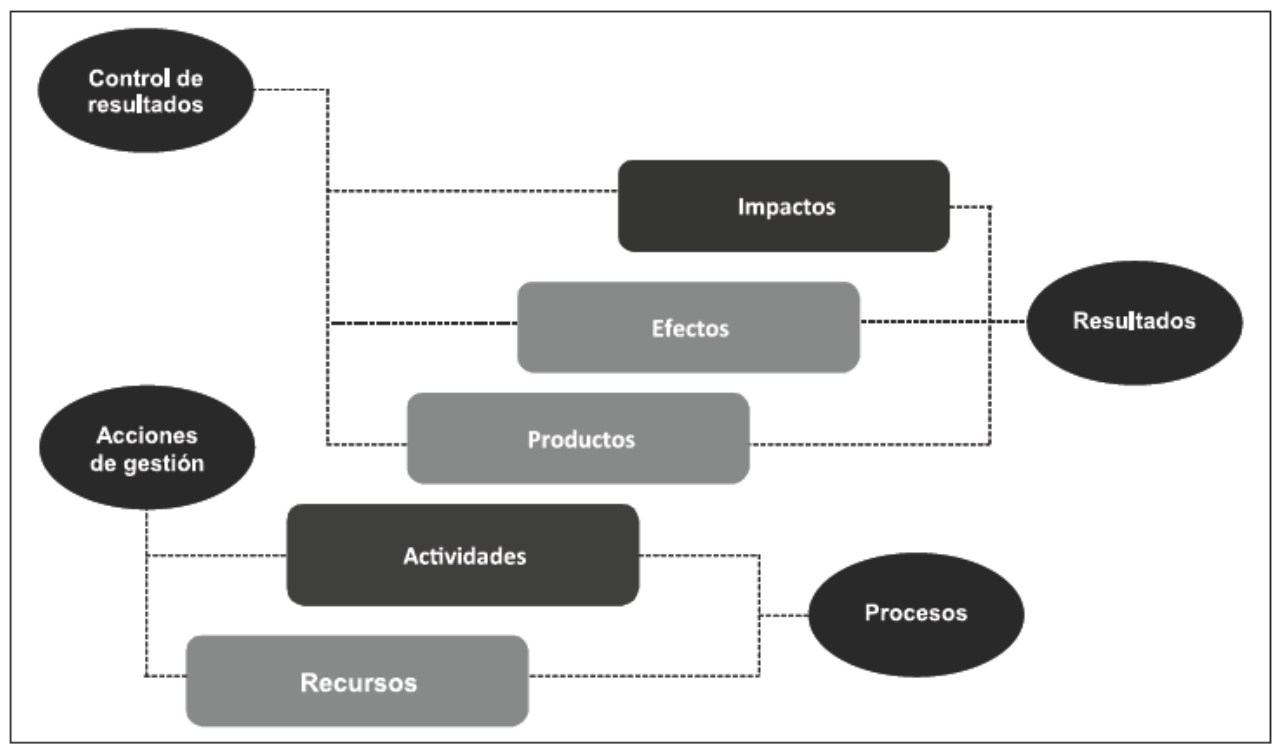




\section{Pensamiento Crítico N. ${ }^{\circ} 3$}

Los resultados de primer nivel se refieren a los efectos directos del proyecto. Corresponden al efecto que los productos generan en las familias, grupos, comunidades o instituciones después de la ejecución de las actividades. Medir los efectos directos significa analizar los cambios habidos en el comportamiento de las familias y los cambios en el desempeño de la población beneficiaria e instituciones. Estos cambios son muy específicos en cada contexto y dependen de las características y objetivos del proyecto. Se puede recurrir a diversos métodos para medir esos cambios: estudios, enfoques participativos, encuestas basadas en cuestionarios, debates temáticos en grupos, etc. En el marco de una evaluación ex post, los indicadores de producto pueden ayudar a evaluar el nivel de eficacia física y financiera de los proyectos.

Los resultados de segundo nivel se refieren a los indicadores de propósito o indicadores de efecto. En el marco de una evaluación ex post, los indicadores de efecto pueden facilitar el análisis de eficacia e impacto. Los resultados de segundo nivel se pueden medir mediante una combinación flexible de métodos pertinentes para cada proyecto, en función del contexto local, regional y nacional. Se pueden utilizar datos e información, tanto de carácter cualitativo, como de carácter cuantitativo.

Los resultados de tercer nivel corresponden al impacto del proyecto. En el marco de una evaluación ex post, se utilizan ciertos indicadores clave en el contexto de la estrategia de desarrollo del país, tales como, por ejemplo, la tasa de incidencia de la pobreza, equidad social y equidad de género. Estos indicadores se pueden complementar con otros, tales como el incremento de la calidad de vida y el mejoramiento de la gobernabilidad del país; de este modo, ayudar a evaluar la pertinencia y el impacto de los proyectos. 


\section{David Medianero Burga}

\section{Terminología de la cadena de resultados}

- Insumos. Recursos financieros, humanos y materiales necesarios para realizar las actividades y conseguir los productos previstos de un proyecto. Pueden ser expresados únicamente como metas financieras o costos.

- Actividades. Medidas adoptadas o tareas realizadas para conseguir un producto específico empleando los recursos asignados.

- Productos. Resultados inmediatos, tangibles o intangibles, que se producen gracias a la ejecución de las actividades.

- Efectos. Resultados generados por los productos entregados por el proyecto en el bienestar de la población beneficiaria.

- Impactos. Resultados positivos y negativos a largo plazo producidos por un proyecto en el bienestar de la población beneficiaria y/o el país.

\section{Criterios de evaluación}

Todo proyecto se realiza con algún objetivo. La evaluación de resultados permite determinar en qué grado se logra el objetivo o cambio deseado. Generalmente, se busca un cambio en las condiciones de vida de la población, en términos de ingresos, acceso a servicios o ejercicio de derechos políticos. Este tipo de evaluación busca determinar la medida en la que se ha logrado el resultado deseado en la población objetivo. Al evaluar los resultados de un proyecto, a fin de determinar si el diseño y la gerencia del mismo fueron adecuados, se ponen énfasis en cinco aspectos importantes:

- Pertinencia. Grado en el cual el objetivo del proyecto es consistente con las prioridades de desarrollo de la población y las políticas de la entidad ejecutora y el organismo financiador.

- Eficacia. Grado en el cual se logran las metas físicas y financieras de un proyecto. En general, la eficacia es la verificación del cumplimiento de los objetivos de una intervención. 


\section{Pensamiento Crítico N. 13}

- Eficiencia. Análisis de los resultados con relación al costo; es decir, análisis de la transformación de los insumos en productos mediante la realización de determinadas actividades.

- Impacto. Valoración socioeconómica global, incluyendo los efectos positivos y negativos, tanto aquellos que son deseados y estaban previstos, como los no previstos y no deseados.

- Sostenibilidad. Apreciación de la capacidad para mantener los impactos positivos del proyecto por un largo periodo de tiempo. Análisis sobre si el efecto global, en términos, por ejemplo, la reducción de la pobreza, es positivo también en el largo plazo.

Estos criterios están estrechamente relacionados al enfoque del marco lógico. Así, la pertinencia alude a la relación del propósito con las prioridades de desarrollo; la eficacia a la relación entre los productos y el propósito; la eficiencia es una comparación de los insumos con los productos; el impacto establece relaciones entre las variables controlables por el proyecto y el fin; y, por último, la sostenibilidad analiza la interacción entre el fin y los supuestos al mismo nivel. Cabe reiterar que en la evaluación ex-post se suele convenir que el concepto de resultado abarca tres niveles de la columna de objetivos del marco lógico: productos (o componentes), efectos (propósito) e impactos (fin). En ocasiones se suele diferenciar a la evaluación de resultados del análisis de impactos con la intención de estimar cualitativa y cuantitativamente la incidencia del proyecto sobre determinados aspectos o áreas del desarrollo. A este análisis se le denomina evaluación de impacto. Para medir los resultados (detectar un cambio), idealmente es necesario contar con datos sobre la población beneficiaria antes y después de la intervención. Alternativamente, es posible fijar una meta ideal y compararla con la meta realmente lograda al final de la ejecución del proyecto. Sin embargo, estos cinco criterios son de carácter general y, si bien constituyen la base fundamental sobre la cual se aborda la presente evaluación, deben ser operativizados a través de la recopilación de información sobre los indicadores correspondientes.

\section{Análisis de pertinencia}

Por pertinencia se entiende la apreciación global sobre si el proyecto se condice con los objetivos de desarrollo del país, así como con las necesidades y prioridades 


\section{David Medianero Burga}

locales. Permite contar con una referencia sólida para gobernantes y funcionarios públicos acerca de la continuación, reformulación o finalización de una intervención. La pertinencia es un tema relevante dentro de la evaluación y debe estar presente desde las etapas iniciales de planificación y a lo largo de todo el ciclo del proyecto.

El análisis de pertinencia es una cuestión de dirección del proyecto con relación a con su propósito. Por otro lado, implica observar los cambios sociales e institucionales que puedan haber ocurrido durante la ejecución del proyecto y evaluar hasta qué punto esto puede alterar la lógica del Programa. Cabe indicar que determinados cambios en las condiciones externas podrían disminuir el grado de prioridad del proyecto o erosionar su fundamento lógico. Por ello, el análisis de pertinencia implica decisiones del más alto nivel sobre la conclusión o continuación de los proyectos y, en este último caso, sobre posibles cambios en la estrategia y metodologías de ejecución. En el contexto del Programa materia de evaluación, el análisis de pertinencia fue orientado de modo que se pudiera verificar la utilización de los recursos en la atención a las poblaciones en situación de pobreza, e implicó realizar las acciones siguientes:

\section{Análisis de pertinencia}

- Análisis de los niveles de pobreza de las zonas de intervención del Programa.

- Análisis de los niveles de desarrollo de la infraestructura en las zonas de intervención del Programa.

- Análisis de la vigencia de las modalidades de ejecución del Programa.

\section{Análisis de eficacia}

El análisis de eficacia de un proyecto consiste en la medición del grado de avance en el logro de sus objetivos y metas, o bien de la probabilidad de que se logre al término del periodo previsto de ejecución. La eficacia implica una apreciación del grado en que se logra el propósito o se espera alcanzarlo. Establecer la eficacia es parte esencial de la evaluación y la información necesaria para llevarla a efecto es provista por las unidades 


\section{Pensamiento Crítico N. ${ }^{\circ} 3$}

ejecutoras correspondientes o, alternativamente, por la información de los sistemas fiscales, tales como el SIAF.

La determinación de la eficacia presupone que el propósito del proyecto ha sido definido operacionalmente y de manera no ambigua, de forma tal que es posible una verificación razonablemente válida. Sin embargo, con frecuencia no se da esto: los objetivos pueden estar formulados de manera poco clara y ser demasiado generales. En tales casos se debe resaltar esa debilidad; el equipo de evaluación tiene que operacionalizar los objetivos sobre la base de la documentación existente del proyecto, para determinar qué se ha logrado realmente.

Usualmente, un proyecto de inversión pública es sólo uno de los muchos factores que pueden contribuir al logro de los objetivos de desarrollo de un país. Por lo tanto, el equipo de evaluación ha fijado su atención sobre las causas de la eficacia (o de la falta de ésta), especialmente en qué grado se han alcanzado los productos esperados, y si estos son suficientes o si se deben hacer cambios en el diseño del proyecto para asegurar un nivel óptimo de eficacia en un tiempo determinado. Sin embargo, es igualmente importante determinar minuciosamente los factores externos (supuestos) que también influyen sobre las probabilidades de éxito del proyecto. En el contexto del Programa materia de evaluación, el análisis de eficacia, tomando como base la información del SIAF, implicó realizar las acciones siguientes:

\section{Análisis de eficacia}

- Comparación de las metas físicas programadas con las metas físicas ejecutadas.

- Comparación de las metas financieras programadas con las metas financieras ejecutadas.

- Verificación de la calidad de los productos generados, a través de intercambios de información y entrevistas con el personal de las unidades ejecutoras. 


\section{David Medianero Burga}

\section{Análisis de eficiencia}

Por eficiencia se entiende el grado en el que los resultados del proyecto se han logrado mediante el uso óptimo de los recursos. Asimismo, el análisis de eficiencia comprende la evaluación de la calidad de la ejecución del presupuesto asignado al proyecto. En otras palabras, el análisis de eficiencia consiste en determinar el grado en el que los diversos insumos se convierten en productos de manera económica. De allí que una forma universal de operativizar el análisis de eficiencia sea a través del cálculo de la ratio costo-efectividad.

La eficiencia es, por otra parte, una forma de expresar la productividad del proceso de implementación; esto es, el grado en que los productos logrados derivan de un uso eficiente de los recursos financieros, humanos y materiales. La mayor dificultad para medir la eficiencia radica en determinar el estándar de comparación o línea de corte. Esto dependerá del tipo de proyecto, de las circunstancias locales y de otros factores. El estándar difícilmente está predeterminado. Por lo tanto, en la práctica depende del equipo de evaluación especificar contra qué estándar se va a medir.

En otras palabras, el análisis de eficiencia en su forma operativa de medición de productividad consiste en establecer una relación cuantitativa entre un indicador de resultado (como, por ejemplo, el número de beneficiarios o el quantum de productos entregados a la población) y un indicador de los costos o insumos utilizados por el proyecto. En el contexto de la presente evaluación, este ha sido el enfoque adoptado, e implico la realización de las tareas siguientes:

\section{Análisis de eficiencia}

- Estimación de la eficacia física, en tanto ésta representa un indicador de resultado.

- Estimación de la eficacia financiera, en tanto ésta representa un indicador de insumo (costos) de un proyecto.

- Relación entre ambos indicadores, a fin de tener una aproximación al concepto de eficiencia o productividad. 


\section{Pensamiento Crítico N. ${ }^{\circ} 3$}

\section{Análisis de impacto}

El impacto de un proyecto son las consecuencias que éste genera en la sociedad, tanto las previstas como las no esperadas, sean positivas o negativas. Para ello, se debe tomar como punto de partida el fin y el propósito del proyecto, pero este proceso va mucho más allá de establecer si los resultados se han logrado. Este tipo de evaluación con frecuencia requiere de investigaciones que abarcan un mayor campo de estudio. La eficacia es un criterio de evaluación estrecho, ya que el propósito del proyecto indica solamente los efectos positivos esperados por las partes involucradas. A diferencia de esto, el concepto de impacto es mucho más amplio porque incluye las consecuencias positivas y negativas, tanto las previstas como las no esperadas. En la determinación de impactos el punto de partida debe ser el propósito del proyecto en la forma en que ha sido definido, así como el grado de eficacia alcanzado, y luego se debe analizar que otros efectos (positivos o negativos, previstos o no esperados) se han producido como resultado de la ejecución del proyecto.

El impacto total es el resultado de condiciones causales complejas que pueden ser difíciles de analizar. Puede ser especialmente problemático probar que los cambios observables son producidos por un determinado proyecto o acción de desarrollo. A fin de comprender las conexiones causales, el evaluador puede necesitar emplear métodos orientados al proceso, como la observación de los participantes. En distintos momentos pueden aparecer diferentes impactos. En el trabajo de evaluación se debe poner atención tanto a los impactos de corto plazo como a los de largo plazo. Las evaluaciones de proyectos en implementación igual pueden producir conclusiones valiosas aplicables en el futuro a proyectos de desarrollo similares. En el contexto del Programa materia de evaluación, el análisis de impacto implicó realizar las acciones siguientes:

\section{Análisis de impacto}

- Determinación de los efectos de los proyectos sobre los niveles de pobreza en las zonas de intervención.

- Determinación de los efectos de los proyectos sobre los niveles de desarrollo de la infraestructura económica y social en las zonas de intervención.

- Determinación de la magnitud de la población beneficiaria alcanzada por los proyectos conformantes del Programa. 


\section{David Medianero Burga}

Gráfico 3. Componentes de la evaluación.

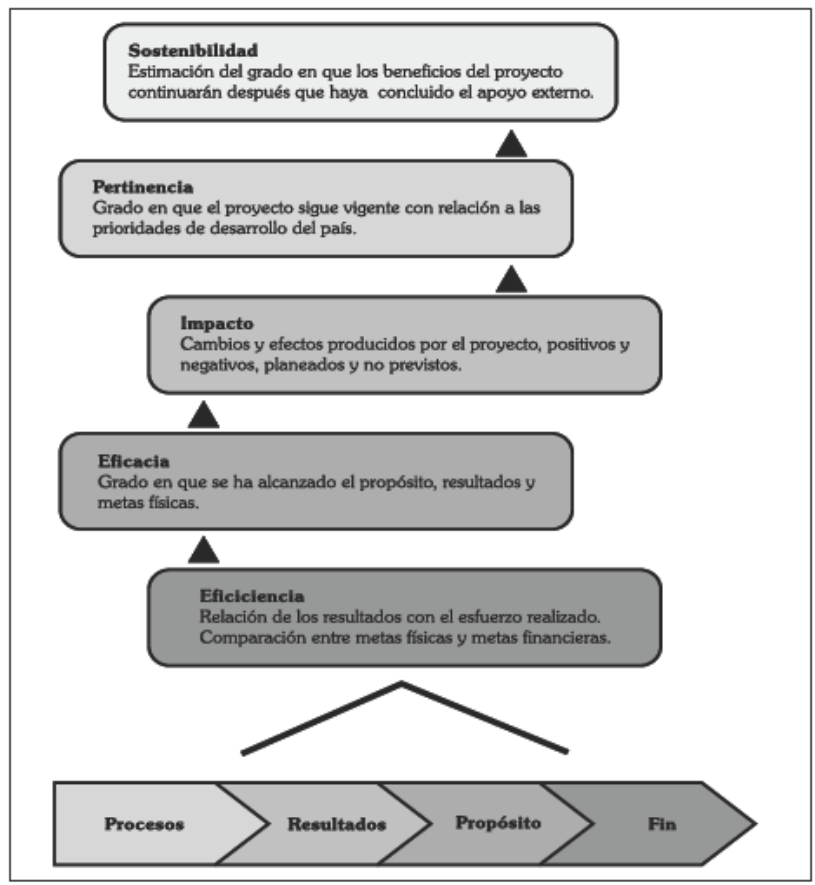

\section{Análisis de sostenibilidad}

La sostenibilidad es una estimación global sobre el grado en que los cambios positivos producidos por los proyectos del Programa continuarán aún después que éstos hayan concluido. Según la $\mathrm{OCDE}^{1}$, la sostenibilidad es la "prueba final" del éxito de un proyecto de desarrollo. Así, la determinación de la sostenibilidad de un proyecto de desarrollo implica mucho más que la respuesta a la pregunta sobre el logro de los objetivos fijados: indica la probabilidad que los impactos positivos continúen una vez que hayan concluido las actividades del proyecto. En el contexto de la presente evaluación, la sostenibilidad se define como la capacidad para generar beneficios duraderos, que permanezcan vigentes incluso después de que el proyecto haya concluido ${ }^{2}$. Los puntos clave a destacar en la definición de sostenibilidad son los siguientes:

1 Organización para la Cooperación y Desarrollo Económico.

2 Véase, Quality Assurance Group PIA/OPRE, Promoting Practical Sustainability. Australian Agency for International Development (AusAID), Canberra, September 2000. 


\section{Pensamiento Crítico N. 13}

- La atención se centra en mantener el flujo de beneficios en lugar del mantenimiento de la intervención misma. Los proyectos, por definición, no son sostenibles, ya que son acciones de carácter temporal, con fechas de inicio y término predeterminadas en los correspondientes estudios de pre inversión y los arreglos institucionales de financiación.

- El concepto de beneficio sostenible, por lo tanto, no necesariamente significa la continuidad de las actividades del proyecto, más allá de un periodo razonable de tiempo.

El análisis de sostenibilidad es, pues, un proceso encaminado a maximizar y dar continuidad al flujo de beneficios para la población. Debe ser un proceso permanente y debe ser actualizado según las cambiantes circunstancias del entorno. Cabe destacar, sin embargo, que no existe una única manera de lograr la sostenibilidad, sino que cada proyecto debe definir su propia estrategia de sostenibilidad, según las circunstancias específicas internas y externas. El análisis de sostenibilidad de un proyecto consiste, pues, en determinar si más allá del logro de sus objetivos, es posible que los impactos positivos continúen después que el proyecto haya concluido. En el contexto del Programa materia de evaluación, el análisis de sostenibilidad ha implicado realizar las acciones siguientes:

\section{Análisis de sostenibilidad}

- Verificación de la capacidad de las unidades ejecutoras para dar continuidad a la atención de los usuarios.

- Verificación de las capacidades sociales e institucionales en las zonas de intervención para administrar los servicios que emergerán con las obras de infraestructura construidas $y / 0$ capacidades instaladas.

- Verificación de capacidades nacionales, especialmente fiscales, para cubrir los costos operativos de los servicios relacionados a los proyectos ejecutados.

En general, en el marco de la presente evaluación se ha recopilado información cualitativa y cuantitativa sobre la ejecución de los proyectos. A partir de dicha ejecución 


\section{David Medianero Burga}

se han efectuado una serie de análisis, con la finalidad de transformar la masa de datos obtenida en información real para la toma de decisiones.

\section{4. Índice global de desempeño}

Como ya ha sido señalado, el objetivo del estudio fue determinar la calidad del programa de proyectos financiado por la CAF, a través de cinco criterios de evaluación: pertinencia, eficacia, eficiencia, impacto y sostenibilidad. Desde esta perspectiva, el desempeño de cada proyecto está determinado por su desenvolvimiento en cada uno de estos enfoques. Sin embargo, a los efectos de facilitar la interpretación de los resultados de la evaluación se construyó un índice a través del cual son sintetizados los resultados del análisis del conjunto de criterios de evaluación. A dicho instrumento se le ha dado el nombre de Índice de Desempeño Global (IDG, en adelante), el cual sintetiza los cinco criterios de la evaluación.

El IDG es una medida de resumen del desempeño de cada uno de los proyectos de la muestra del Programa. Por lo tanto, constituye el resumen de la aplicación de los cinco criterios de evaluación. Mide en forma general el aporte de las inversiones realizadas al desarrollo del país, particularmente al logro del objetivo esencial del Programa: disminuir los niveles de pobreza mediante la dotación de infraestructura económico-social en los territorios de menor desarrollo relativo del país.

Para calcular el IDG fue necesario crear un índice para cada uno de los criterios de evaluación, dando lugar a los siguientes índices: índice de pertinencia, índice de eficacia física, índice de eficacia financiera, índice de productividad (como representación del criterio de eficacia), índice de impacto e índice de sostenibilidad. En todos los casos, se ha utilizado la información estadística más actualizada posible. Todos los indicadores se basan en información estadística cuantitativa, a excepción del índice de sostenibilidad, el cual se ha construido sobre la base de la opinión de los responsables de las unidades ejecutoras correspondientes. En todos los casos se ha buscado expresar el índice en una escala de 1 a 10, que facilite la comparabilidad entre si y, por ende, la construcción del ranking de proyectos en función del desempeño global. 


\section{Pensamiento Crítico N. ${ }^{\circ} 3$}

\section{Indicadores utilizados}

Cabe destacar que los indicadores utilizados son de dos tipos: indicadores cuantitativos (índices de pertinencia, eficacia física, eficacia financiera, eficiencia e impacto), $e$ indicadores cualitativos (índice de sostenibilidad, basado en la encuesta realizada). A su vez, cada indicador puede englobar una o más variables. A continuación se describen los indicadores y variables utilizados en la construcción del IDG.

- Pertinencia. La aplicación del criterio de pertinencia implicó el uso de dos indicadores: tasa de pobreza y nivel de infraestructura. La tasa de pobreza utilizada refleja el nivel de pobreza que se presenta en el ámbito de ejecución de cada proyecto para el año $2007^{3}$, periodo anterior a la ejecución del Programa. Asimismo, el stock de infraestructura mide el desarrollo del nivel de infraestructura en el ámbito de ejecución de cada proyecto al año $2007^{4}$.

- Eficacia física. Para operativizar el criterio de eficacia física se toma como referencia el índice de eficacia física, calculado en unidades porcentuales, el cual expresa el grado de cumplimiento de las metas físicas establecidas en cada proyecto ${ }^{5}$.

- Eficacia financiera. Para operativizar el criterio de eficacia financiera se toma como referencia el índice de eficacia financiera, calculado en unidades porcentuales, el cual expresa el grado de cumplimiento de las metas financieras establecidas en cada proyecto.

- Eficiencia. El criterio de eficiencia ha sido operativizado mediante la fórmula de productividad, relacionando los valores representativos de las metas físicas con los de las metas financieras.

3 En este caso, se calculó una tasa de pobreza promedio para cada proyecto en función a tasas de pobreza distrital 2007 correspondiente a su ámbito de ejecución. Dichos cálculos se basaron en los resultados de los Censos de Población y Vivienda 2007, elaborado por el INEI.

4 Para ello, se tomó como referencia el índice de infraestructura a nivel departamental, elaborado por el Consejo Nacional de Competitividad (CNC) al año 2007. Se toma a dicha variable como proxy del nivel de desarrollo que se presenta en el ámbito de cada proyecto.

5 Cabe mencionar que, los datos utilizados para calcular el nivel de avance físico y financiero alcanzado por cada proyecto provienen de dos fuentes de información: la encuesta realizada a las 31 unidades ejecutoras que comprende la muestra y la revisión de la base de datos del Sistema Integrado de Administración Financiera (SIAF) del MEF. 


\section{David Medianero Burga}

- Impacto. La aplicación del análisis de impacto se realizó recurriendo al diferencial de las tasas de pobreza 2007 y 2008 , a nivel departamental. Ha sido imposible que este análisis se realice a nivel distrital, debido al hecho de que el INEI, la fuente oficial de este tópico, sólo publica cifras de pobreza a nivel regional para el $2008{ }^{6}$ Dichas variables se expresan en unidades porcentuales.

- Sostenibilidad. La sostenibilidad ha sido medida a través de un índice de sostenibilidad global construido en el contexto del presente estudio ${ }^{7}$. Este índice comprende a su vez tres dimensiones de sostenibilidad: general, social y económico-financiera. La sostenibilidad general se refiere a la permanencia de los beneficios directos de los proyectos. La sostenibilidad social se refiere a los cambios en el mejoramiento de la calidad de vida de la población beneficiaria. Por último, la sostenibilidad económico-financiera se refiere al desempeño financiero de los proyectos en el futuro cercano.

Gráfico 4. Metodología del índice de desempeño global.

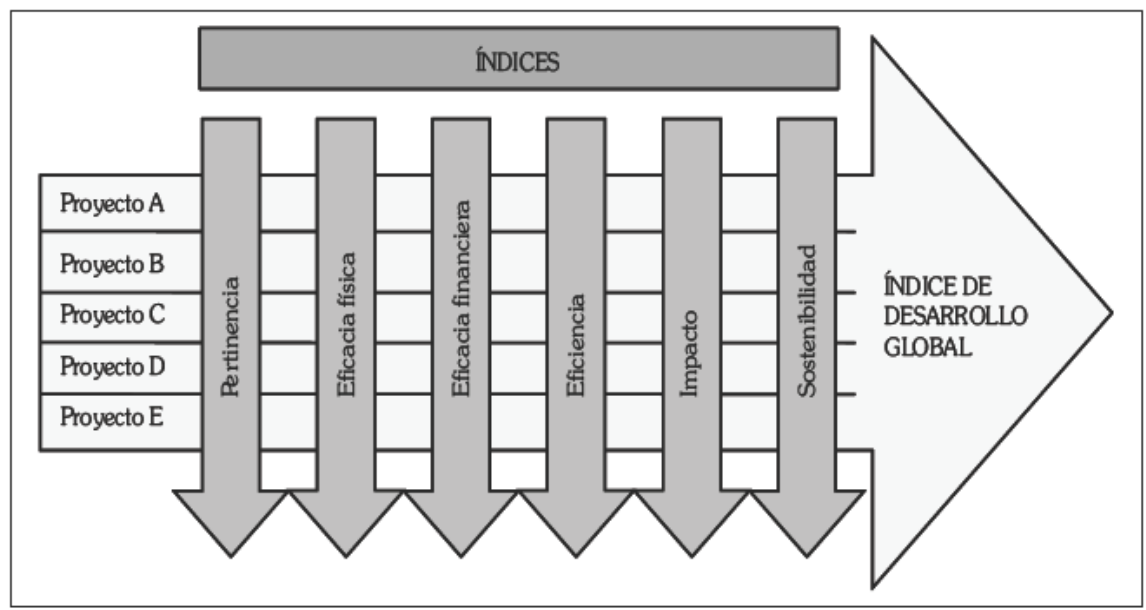

6 El indicador global de desempeño utiliza, en el criterio de impacto, el diferencial de tasa de pobreza departamental. Sin embargo, también se ha realizado una evaluación de impacto a nivel distrital. Para ello, se tomó en 2007 la tasa de pobreza promedio calculada en el análisis de pertinencia, y para el 2008, se utilizó como variable proxy de la tasa de pobreza distrital a la tasa de pobreza departamental 2008. A pesar de las limitaciones metodológicas del caso, se optó por utilizar el análisis de impacto departamental. Cabe indicar que, ambas metodologías muestran resultados similares.

7 El índice se basa en los resultados obtenidos de la encuesta realizada a las diferentes unidades ejecutoras (31). 


\section{Pensamiento Crítico N. 13}

Con base en ello, se jerarquizaron los proyectos de acuerdo al desempeño global, dando lugar a un ranking de proyectos en función del IDG. El índice al ser una medida de los proyectos sobre una escala de 0 a 1, permite obtener, asimismo, la calificación promedio del Programa en su conjunto. Una calificación mayor al 0,5 estaría indicando un desempeño positivo del Programa, y en la medida que el puntaje promedio suba, esto debería interpretarse como un mejoramiento del desempeño de la cartera en su conjunto. En cambio, una calificación promedio menor al 0,5 significaría un bajo desempeño global del Programa.

\section{Estandarización de valores}

A fin de hacer comparables todas las medidas relacionadas a los criterios de evaluación, se ha realizado un trabajo de estandarización de valores. A tal efecto, se ha utilizado dos metodologías: el método de los valores límite y el método de puntuaciones z. Finalmente, se optó por los resultados que emergen del primero de los métodos mencionados.

Método de valores límite. El procedimiento de construcción de cada índice ha implicado la realización de tres pasos: (a) identificación de indicadores válidos y de la fuente de información correspondiente; (b) determinación de los valores mínimos y máximos, llamados genéricamente valores límite, para cada uno de los indicadores (en el caso de los índices de eficacia física, eficacia financiera y eficiencia esto no ha sido necesario ya que de modo natural están expresados en tanto por uno); (c) Cálculo del índice de cada proyecto, mediante la aplicación de la formula siguiente:

$$
\text { Índice }=\frac{\text { valor real - valor mínimo }}{\text { valor máximo - valor mínimo }}
$$

El desempeño de cada proyecto desde cualquiera de los criterios de evaluación, se expresa como valor entre 0 y 1 . Sobre esta base se procedió al cálculo del IDG como simple promedio de los índices de los componentes. Finalmente, los proyectos fueron ordenados de mayor a menor, en función del valor de sus respectivos índices agregados. 


\section{David Medianero Burga}

El Gráfico 5 muestra la escala de medición de cada uno de los índices.

Gráfico 5. Construcción de índices.

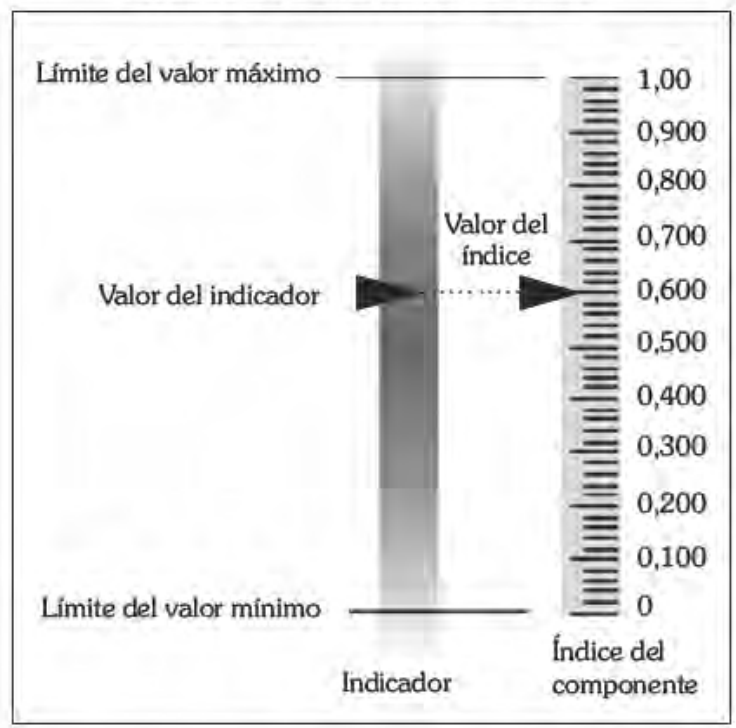

Método de puntuaciones $\mathbf{z}$. Alternativamente, se ha construido un ranking de proyectos mediante la aplicación de la metodología estadística conocida como puntuaciones $\mathrm{z}$. Las puntuaciones $\mathrm{z}$ son transformaciones que se pueden hacer a los valores o las puntuaciones obtenidas, con el propósito de analizar su distancia respecto a la media, en unidades de desviación estándar. Una puntuación z nos indica la dirección y el grado en que un valor individual obtenido se aleja de la media, en una escala de desviación estándar. Las puntuaciones z constituyen el método más comúnmente utilizado para estandarizar la escala de una variable medida en un nivel de intervalos ${ }^{8}$.

Dicha metodología permite uniformizar las variables o indicadores representativos de cada criterio y formar un sólo índice, el cual, a su vez, puede permitir jerarquizar el desempeño de los proyectos dentro del Programa en una suerte de ranking de los mismos.

8 Véase: Metodología de la investigación, Roberto Hernández y otro. Mc Graw Hill, México 2006. 


\section{Pensamiento Crítico N. ${ }^{\circ}$ I3}

\section{Fuentes de información}

Las fuentes de información utilizadas para la operativización del cálculo de los indicadores han sido tanto de orden primario como secundario. La fuente primaria es la encuesta realizada en el marco del presente estudio, en tanto que las principales fuentes secundarias han sido: Ministerio de Economía y Finanzas (SIAF y Banco de Proyectos), INEI (Perfil de Pobreza 2004-2008 y otros informes de pobreza). Los datos de infraestructura se han obtenido de Perú Compite, un órgano de la Presidencia del Consejo de Ministros.

Gráfico 6. Criterios de evaluación y fuentes de información.

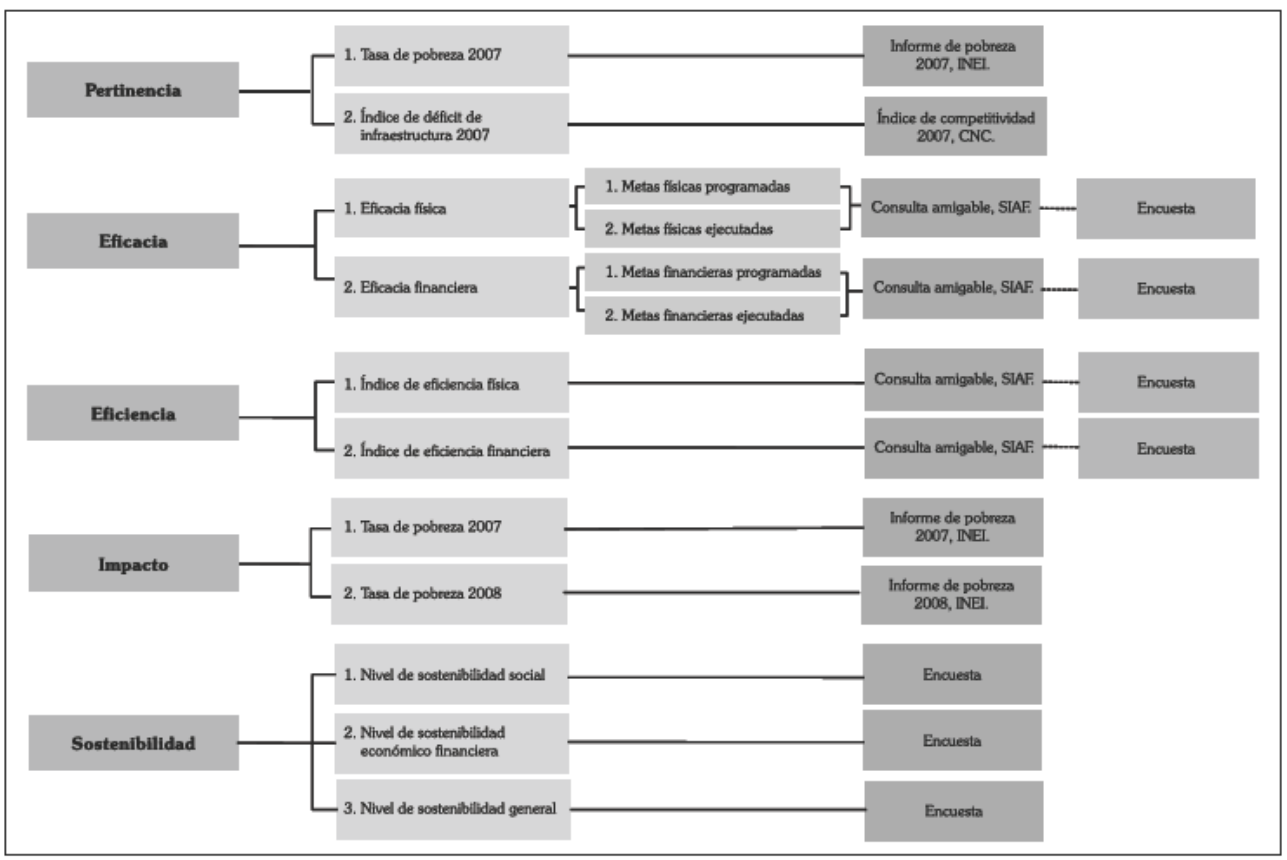

